\title{
Iron, anemia and hepcidin in malaria
}

\author{
Natasha Spottiswoode ${ }^{1,2}$, Patrick E. Duffy ${ }^{1}$ and Hal Drakesmith ${ }^{2}$ * \\ ${ }^{1}$ Laboratory of Malaria Immunology and Vaccinology, National Institute of Allergy and Infectious Diseases, National Institutes of Health, Bethesda, MD, USA \\ ${ }^{2}$ MRC Human Immunology Unit, Weatherall Institute of Molecular Medicine, University of Oxford, Oxford, UK
}

\section{Edited by:}

Raffaella Gozzelino, Instituto

Gulbenkian de Ciência, Portugal

\section{Reviewed by:}

Carla Cerami, University of North Carolina at Chapel Hill, USA

Aubrey Cunnington, Imperial College London, UK

\section{*Correspondence:}

Hal Drakesmith, MRC Human Immunology Unit, Weatherall Institute of Molecular Medicine, University of Oxford, Oxford, OX3 9DS, UK e-mail:hdrakes@hammer. imm.ox.ac.uk
Malaria and iron have a complex but important relationship. Plasmodium proliferation requires iron, both during the clinically silent liver stage of growth and in the diseaseassociated phase of erythrocyte infection. Precisely how the protozoan acquires its iron from its mammalian host remains unclear, but iron chelators can inhibit pathogen growth in vitro and in animal models. In humans, iron deficiency appears to protect against severe malaria, while iron supplementation increases risks of infection and disease. Malaria itself causes profound disturbances in physiological iron distribution and utilization, through mechanisms that include hemolysis, release of heme, dyserythropoiesis, anemia, deposition of iron in macrophages, and inhibition of dietary iron absorption. These effects have significant consequences. Malarial anemia is a major global health problem, especially in children, that remains incompletely understood and is not straightforward to treat. Furthermore, the changes in iron metabolism during a malaria infection may modulate susceptibility to co-infections. The release of heme and accumulation of iron in granulocytes may explain increased vulnerability to non-typhoidal Salmonella during malaria. The redistribution of iron away from hepatocytes and into macrophages may confer host resistance to superinfection, whereby blood-stage parasitemia prevents the development of a second liver-stage Plasmodium infection in the same organism. Key to understanding the pathophysiology of iron metabolism in malaria is the activity of the iron regulatory hormone hepcidin. Hepcidin is upregulated during blood-stage parasitemia and likely mediates much of the iron redistribution that accompanies disease. Understanding the regulation and role of hepcidin may offer new opportunities to combat malaria and formulate better approaches to treat anemia in the developing world.

Keywords: hepcidin, malaria, iron, anemia, global health

\section{INTRODUCTION}

Among the many nutrients required for human survival, iron plays a unique role in determining disease susceptibility. This relationship is especially well studied in the case of malaria. Parasites of the genus Plasmodium cause malaria, although "malaria" is generally used to refer to symptomatic Plasmodium falciparum infection, unless otherwise specified. Malaria is currently one of the most geographically widespread and deadly diseases, and responsible for the deaths of an estimated 600,000 people per year (Malaria World Report, $2013^{1}$ ). The controversial relationships between malaria and iron have been the subject of widespread discussion and debate by the global health community since 2006, when a randomized large-scale trial on the island of Pemba found that iron supplementation in children was linked with an increase in malaria-related mortality (Sazawal et al., 2006). Two Cochrane reviews (Ojukwu et al., 2009; Okebe et al., 2011) published subsequent to this finding have not resolved this conundrum for physicians and policymakers. Furthermore, while iron supplementation appears to be linked with increased malarial mortality, malaria infections are a major global cause of anemia (Kassebaum et al., 2014); and measures taken to decrease malaria at a population level also decrease anemia (Meremikwu et al., 2012).

\footnotetext{
${ }^{1}$ http://www.who.int/malaria/publications/world_malaria_report_2013/en/
}

The interactions between malaria and iron have only lately begun to be understood at the molecular level. Primarily, the discovery of the iron regulatory hormone hepcidin has given us new understanding of human iron physiology and pathophysiology. Hepcidin serves to block iron absorption from the diet and also to route iron in the body into macrophages and away from the serum. Hepcidin plays a complex but vital role in both the iron restriction that occurs during malaria infection, and in determining iron status and thereby influencing disease susceptibility. In this review, we examine the known interactions between physiological iron deficiency or repletion, iron supplementation, malaria, and hepcidin, and offer recommendations and suggestions for future work.

\section{IRON DEFICIENCY PROTECTS FROM MALARIA INFECTION}

Variations in the iron levels of susceptible hosts may modulate the frequency and clinical severity of malaria infections. Gwamaka et al. (2012) collected detailed data from a large cohort of Tanzanian children (birth -3 years). In this vulnerable population, iron deficiency at healthy aparasitemic visits was strongly associated with decreased future risk of parasitemia and severe malaria (Gwamaka et al., 2012). Iron deficiency in this study was defined by low ferritin $(<30 \mathrm{ng} / \mathrm{mL})$ in individuals with low C-reactive protein (CRP). A higher ferritin cutoff $(<70 \mathrm{ng} / \mathrm{mL})$ was used to 
define individuals with higher CRP as iron deficient; plasma ferritin is considered to be representative of iron stores in healthy individuals but increases acutely in infections.

A further study in a slightly older cohort of Kenyan children ( 8 months- 8 years) found that iron repletion (defined as ferritin $\geq 12 \mathrm{ng} / \mathrm{mL}$, with transferrin saturation $\geq 10 \%$, children with high CRP excluded), was predictive of clinical malaria episodes in the year following measurement (Nyakeriga et al., 2004). Separately, Jonker et al. (2012b) noted a similar effect in Malawian children (6 months-5 years): iron-deficient children (defined as plasma ferritin $<30 \mathrm{ng} / \mathrm{mL}$ ), had a lower incidence of clinical malaria the subsequent year.

Studies performed in pregnant women also may indicate an association between iron deficiency and protection from clinical malaria, although somewhat less data are available. Two crosssectional studies have found that at the time of delivery, placental malaria was associated with iron replete status (Kabyemela et al., 2008; Senga et al., 2011). A limitation of this approach is that all currently used measures of iron status, such as ferritin, can be distorted by inflammation and infection, and thus cross-sectional studies may be of limited utility in understanding this relationship. Only one study has so far attempted to examine the predictive values of iron status on future placental or peripheral parasitemia in pregnant women (Senga et al., 2012). This study measured iron status by examining zinc protoporyphyrin (ZPP) levels. At both the first antenatal visit and at delivery, levels of ZPP indicative of iron repletion were associated with parasitemia; however, analyzing these data for ZPP as a predictive measure were complicated by the elevation of ZPP by concurrent parasitemia. The authors concluded that in this population, with a high incidence of parasitemia and associated inflammation, ZPP alone is not a valid measure of iron status, a concern that also could be applied to pediatric populations. Although the available data suggest a relationship, further studies that recruit women early in pregnancy, stratify carefully by gravidity and malaria transmission intensity, and follow their outcomes closely are required to definitively answer the question of whether iron status in women can predict malaria risk, as in pediatric populations.

\section{IRON SUPPLEMENTATION AND MALARIA INFECTION}

While iron deficiency appears to offer some protection against malaria, iron supplementation may increase the vulnerability of susceptible populations to infection. Consequently, the use of population-scale iron supplementation in malaria-endemic areas is currently highly controversial. Early studies postulated a link between oral intake of iron and malaria susceptibility (Murray et al., 1978), as exemplified by flare-ups of latent infections following refeeding (Murray et al., 1975; Murray and Murray, 1977) or differing malaria susceptibility associated with different diets (Murray et al., 1980). In 2006, the "Pemba trial," a large-scale, randomized trial of iron supplementation in an area with very high malaria transmission, was stopped prematurely when trial monitoring boards found a link between the supplementation of children with iron and folic acid and subsequent malaria infection and mortality (Sazawal et al., 2006). Several studies that followed the Pemba trial produced results that appeared to contradict this finding, showing either no association between supplementation and malaria risk (Desai et al., 2003; Ouedraogo et al., 2008), or a protective effect of iron supplementation (Zlotkin et al., 2013). However, many of the studies that followed the Pemba trial were smaller in scale and/or introduced measures to combat malaria, such as bednets or intermittent preventative chemoprophylaxis, which may have masked any increase in malaria susceptibility.

Two Cochrane reviews have since been published in attempts to reconcile the apparently disparate findings of the Pemba study and subsequent trials (Ojukwu et al., 2009; Okebe et al., 2011). Both reviews concluded that iron supplementation did not increase the risk of malaria infection in children when "regular malaria surveillance and treatment services" were provided. However, many areas of the world have inadequate malaria surveillance and treatment, coupled with high levels of iron deficiency and anemia. We would posit that two major questions remain unanswered by the current literature.

First, to what degree must malaria be controlled, or treatment and prevention practices put in place, before the benefits of giving iron outweigh the risks? This question remains practically difficult to answer due to the ethical difficulties inherent in providing iron supplementation without introducing malariareduction measures, but further guidance is required for clinicians, nutritionists, and policymakers.

A second and related question is: should iron supplementation be restricted to those children who are anemic and/or iron deprived? The updated Cochrane review found that the benefits of iron supplementation were greatest among children who had the lowest hemoglobin levels at baseline (Okebe et al., 2011). Not only might these children reap the greatest benefits from supplementation, they may also be less likely to exhibit increased malaria incidence or severity as a consequence of increased iron stores. In a substudy of the Pemba trial, in which children were more closely monitored and given malaria-reduction measures such as insecticide-treated bednets (ITNs), the authors did not find that iron supplementation associated with increased malaria incidence or mortality (Sazawal et al., 2006). Moreover, children in this substudy who were iron deficient and anemic at baseline showed a significant reduction in adverse events, including malaria episodes, when supplemented with iron and folic acid (Sazawal et al., 2006). This finding was apparently echoed by a subsequent study that examined the effect of food fortification (micronutrient powders with or without iron) on malaria risk in Ghanaian children (Zlotkin et al., 2013), which found that children who were both iron deficient and anemic at baseline showed reduced incidence of malaria following iron supplementation. However, as was mentioned in an editorial on this study (Prentice etal., 2013) in the Ghanian trial, the ironcontaining micronutrient powders were not actually effective at reducing anemia, making these results challenging to interpret. Moreover, iron supplementation in this trial was found to be associated with increased hospital admissions during the intervention period.

Targeting iron supplementation toward iron-deficient or anemic children is complicated by both practical and fiscal obstacles. As previously stated, most indicators of iron status can be confounded by infection or inflammation, limiting their 
interpretation in the field, and the widespread use of such tests may be difficult to fund. A solution to the problem may be to find more efficient ways to avoid giving iron to individuals who are iron replete, infected, or at high risk of infection (Atkinson etal., 2014). For example, in many areas, malaria transmission and prevalence of iron deficiency is seasonal; iron supplementation given outside of the malaria season may be less likely to increase susceptibility to infection, although studies are required to assess whether sub-patent parasitemias during the dry season may be increased by iron supplementation and cause illness.

When considering supplementation guidelines in pregnant women, it should be considered that primigravidae are both more susceptible to malaria infection and less prone to iron deficiency. One study demonstrated that intravenous iron supplementation increased malaria frequency in primigravidae but not multigravidae (Oppenheimer et al., 1986). Studies focusing on the effects of randomized oral iron supplementation have produced differing results (Menendez et al., 1994; Nacher et al., 2003), but relatively few have been performed thus far. In a large cohort study, it was found that iron-replete primigravidae are significantly more likely to experience placental malaria, but this effect loses significance in multigravidae (Kabyemela et al., 2008; Friedman et al., 2009). Summarizing the available evidence, adjusted supplementation guidelines that recommend lower iron supplementation to primigravidae may be advantageous.

\section{HOW THE MALARIA PARASITE BENEFITS FROM IRON}

Iron is a limiting factor for the growth of many bacterial or protozoan pathogens. Iron-chelating agents such as desferrioxamine have been shown to restrict malaria growth in vitro (RaventosSuarez et al., 1982), in murine models of malaria infection (Fritsch et al., 1985; Ferrer et al., 2012), and in malaria-infected monkeys (Pollack et al., 1987) (reviewed in Mabeza etal., 1999). Consequently, iron-chelating agents have been considered as an adjunct or primary antimalarial therapy.

In humans, preliminary work on the use of the iron chelators desferrioxamine, or the orally administered deferiprone, as an adjunct to standard antimalarial therapy seemed promising (Traore et al., 1991). However, after further studies showed no clear benefit from administration of chelators, and one seemed to hint at a slight increase in mortality in the trial group treated with desferrioxamine (Thuma et al., 1998), a Cochrane review recommended that trials testing iron chelators for malaria treatment be discontinued (Smith and Meremikwu, 2003).

The development of antimalarial drugs that target the parasite's access to iron might meet with more success if we had a better understanding of how Plasmodium parasites acquire iron during their various life stages. Blood-stage parasites have been theorized to acquire iron from serum transferrin (Rodriguez and Jungery, 1986), from iron produced during the breakdown of hemoglobin, or from a free pool of intracellular iron (Hershko and Peto, 1988), but this important issue remains unresolved. Very little work has been done that investigates the acquisition of iron by the obligate liver or mosquito life stages of malaria infection; these continue to be fruitful areas for future research. For example, identification of Plasmodium-encoded iron transporters would both increase our understanding of the mechanism by which different life stages obtain their iron, and provide new drug targets aimed at inhibiting parasite growth.

Iron repletion may also have effects on parasite growth through different mechanisms than direct utilization by the parasite. A full synopsis of the literature is beyond the scope of this review, but iron has many effects on the immune system (McDermid and Prentice, 2006). In malaria specifically, it has been suggested that parasitized red blood cells from iron-deficient hosts may be more efficiently phagocytized, based on evidence from a murine model (Matsuzaki-Moriya et al., 2011). Finally, some Plasmodium species, notably $P$. vivax, live preferentially in young reticulocytes. Reticulocyte production can be restricted in highly anemic hosts, and this has been proposed as a mechanistic explanation for a study that showed an inverse association between severe anemia and P. vivax infection (Manning et al., 2012).

\section{HUMAN IRON CONTROL: THE HORMONE HEPCIDIN}

Systemic mammalian iron metabolism is controlled at the level of iron absorbance from the diet and iron recycling through macrophages. Approximately $1 \mathrm{mg}$ of iron is absorbed from the diet every day, roughly equivalent to the iron that is lost daily in poorly regulated activities such as sweating, any bleeding, and the sloughing off of enterocytes. At the same time, iron already in the body is constantly being recycled as macrophages phagocytose senescent or damaged red blood cells, digest the heme and extract the iron they contain, and export that iron back into the circulation.

The export of iron across the basolateral membrane of enterocytes and the recycling of iron through macrophages are dependent on the same transport protein: ferroportin, the sole currently identified mammalian iron export protein (Abboud and Haile, 2000; Donovan et al., 2000; McKie et al., 2000). The release of iron from enterocytes into the bloodstream is the final step of absorption of iron from the diet. Hepcidin, discovered a decade ago by three groups working independently (Krause et al., 2000; Pigeon et al., 2001; Park et al., 2001), is a 25-amino acid protein that binds to ferroportin and causes it to be internalized and degraded (Nemeth et al., 2004). The effect of ferroportin inhibition by hepcidin is therefore to block uptake of dietary iron from the intestine, and to increase the accumulation of iron in macrophages. The result is a decrease in serum iron levels, which routes iron away from pathogens that could potentially exploit circulating iron, but may also render the host anemic by restricting iron availability to the erythron.

Evidence from both animal models and from human genetic lesions suggests that the hepcidin-ferroportin interaction is functionally non-redundant. Mice underexpressing hepcidin are severely iron-overloaded (Nicolas et al., 2001), mimicking the human genetic disorder hereditary hemochromatosis, which (in rare cases) is caused by genetic lesions in the hepcidin gene itself (Roetto et al., 2003), in genes that encode hepcidin-regulatory factors (Bridle et al., 2003), or by mutations in ferroportin that render it resistant to hepcidin control (Drakesmith et al., 2005). Conversely, mice that overexpress hepcidin are fatally anemic 
(Nicolas et al., 2002a), while patients with mutations that lead to chronic hepcidin overexpression suffer from iron-refractory iron-deficiency anemia (Finberg et al., 2008).

Hepcidin increases in response to high iron conditions (Pigeon et al., 2001), but it is also upregulated in response to infectious and inflammatory stimuli (Nicolas et al., 2002b). Hepcidin is controlled homeostatically primarily via the bone morphogenetic protein (BMP) pathway. BMPs signal through the phosphorylation of SMAD transcription factors, which bind to a well-studied site on the hepcidin promoter and increase hepcidin transcription (Wang et al., 2005). Inflammation and infection also induce interleukin (IL)-6 or IL-22, which can upregulate hepcidin through the phosphorylation of STAT3 (Nemeth et al., 2003; Armitage et al., 2011). BMP/SMAD signaling has also recently been linked to hepcidin upregulation in infectious or inflammatory conditions by the molecule activin $\mathrm{B}$, another member of the transforming growth factor $\beta$ (TGF $\beta$ ) superfamily that is upregulated by inflammatory stimuli but acts to upregulate hepcidin through SMAD signaling (Besson-Fournier et al., 2012). Hepcidin is suppressed by anemia, hypoxia, or erythropoietic drive, but, at the time of writing, the pathways underlying hepcidin suppression are less well articulated than are those involved in its upregulation.

\section{UPREGULATION OF HEPCIDIN IN MALARIA: CAUSES AND CONSEOUENCES}

Hepcidin is upregulated in infections by bacterial, fungal, and viral pathogens (Armitage et al., 2011). Multiple studies have found that hepcidin is upregulated in malaria infection in symptomatic and asymptomatic natural human infections (Howard et al., 2007; de Mast et al., 2009a, 2010), in experimentally controlled human infections (de Mast et al., 2009b), and in murine models of malaria infection (Portugal et al., 2011; Wang et al., 2011). Resolution of infection leads to normalization of hepcidin levels (de Mast et al., 2009a).

Hepcidin's upregulation in malaria has several important consequences. First, the upregulation of hepcidin leads to iron accumulation in macrophages and a decrease in serum iron, possibly contributing to the dyserythropoiesis and anemia that can accompany malaria infections. Additionally, hepcidin upregulation directly blocks dietary iron absorption: children with post-malarial anemia have high hepcidin levels and poorly incorporate orally administered iron into their red blood cells (Prentice et al., 2012).

The mechanisms whereby hepcidin is upregulated in malaria infection have yet to be fully characterized. IL-6 has been shown to be correlated with hepcidin in some studies (CasalsPascual etal., 2012; Burte etal., 2013; Jonker et al., 2013), but in another study, urinary IL-6 and hepcidin were not significantly associated after a multiple, stepwise linear regression in infected humans (de Mast et al., 2009a). In one ex vivo study, human peripheral blood mononuclear cells co-incubated with Plasmodium-infected red blood cells showed a significant upregulation of hepcidin mRNA without concomitant IL-6 message increase (Armitage et al., 2009), but the relative contribution of peripheral blood mononuclear cells to systemic hepcidin levels is unknown. Finally, a study looking at the mechanisms by which blood-stage malaria infection can prevent the establishment of a liver-stage infection, which is thought to be modulated by hepcidin, found that liver-stage inhibition was preserved in mice treated with anti-IL-6 antibodies (Portugal et al., 2011). In brief, the role of IL-6 in hepcidin regulation in malaria remains controversial, and it is, as yet, unclear which other pathways may contribute to hepcidin upregulation in malaria infection.

Despite the paucity of knowledge on precisely how hepcidin is upregulated in malaria, the fact that both infection and iron repletion lead to increased hepcidin, and that increased hepcidin prevents oral iron absorption, suggests its potential utility as a point of care test to guide iron supplementation in areas of high infectious burden. Low hepcidin would indicate both the absence of infection and the probability of efficient iron absorption, while high hepcidin would indicate either adequate iron stores or ongoing infection. In the latter situation, iron supplementation would likely be unnecessary due to adequate iron stores, poorly absorbed as a direct result of high hepcidin, and/or potentially harmful if hepcidin is elevated due to an ongoing infection.

\section{UPREGULATION OF HEPCIDIN IN MALARIA: FOCUSING ON CO-INFECTION}

Hepcidin has been recently shown to play a crucial role in determining the multiplicity of malaria infections within a single host. The obligate liver stage of the malaria parasite requires iron: hepcidin peptide injection or hepcidin overexpression by transgene or viral vector can reduce parasite survival at the crucial hepatic bottleneck (Portugal et al., 2011). The hepcidin upregulation initiated by one blood-stage infection thereby blocks the establishment of a second infection (Portugal et al., 2011).

The physiological redistribution of iron as a consequence of hepcidin upregulation may also have a significant effect on host susceptibility to other bacterial, viral, or protozoa parasites (see Figure 1). In a blood-stage malaria infection, raised hepcidin is expected to contribute to increased macrophage iron levels, as does increased erythrophagocytosis. This increase in bioavailable macrophage iron may benefit pathogens that exploit the macrophage niche (van Santen etal., 2013). In particular, hepcidin upregulation may help to explain the association between malaria infections and susceptibility to non-typhoid salmonella (NTS). The epidemiological link between malaria and NTS is well established (Mabey et al., 1987). Iron has been implicated in the contribution of malaria to NTS susceptibility through increases in both free heme and heme-oxygenase expression (Cunnington et al., 2012). By routing iron to accumulate in macrophages, the hepcidin response to malaria may also render the host more vulnerable to NTS directly (van Santen et al., 2013). Similarly, tuberculosis could conceivably benefit from the increased iron availability in its macrophage niche, but the specific role of iron and hepcidin in this important co-infection has not been examined at the time of writing.

An important question is whether malaria-induced alteration of iron metabolism may modulate susceptibility to viral infections and vice versa. Malaria infections are associated with 


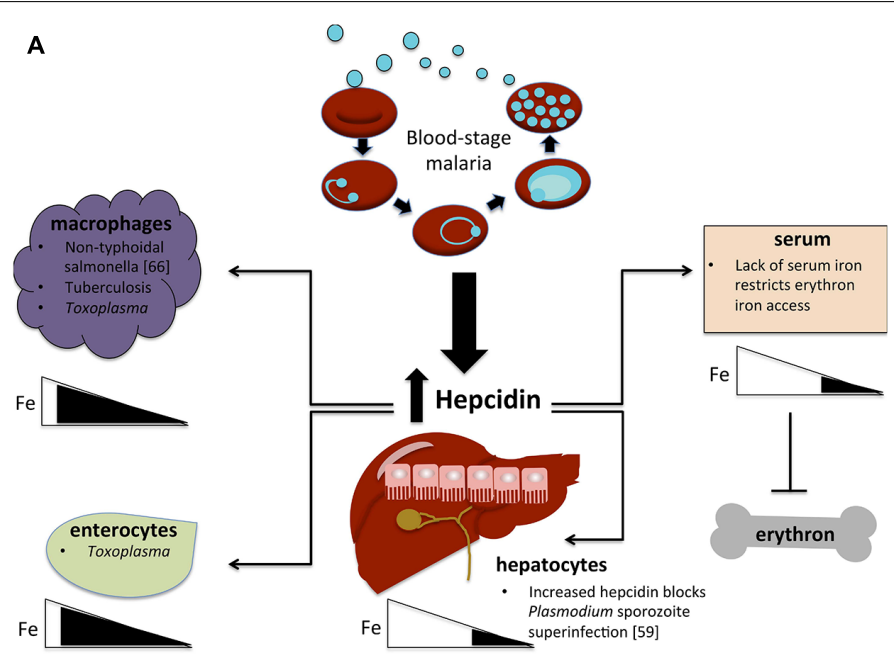

B

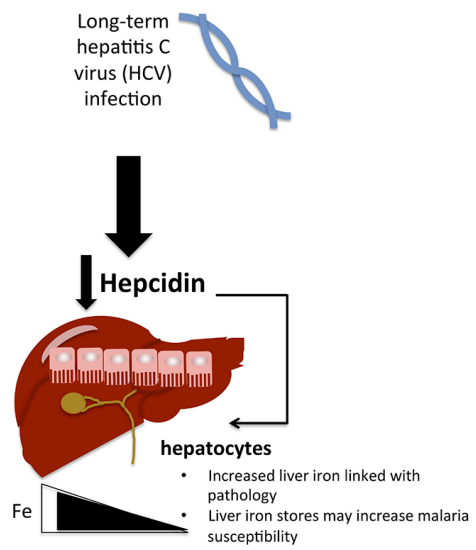

C

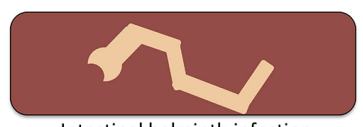

Intestinal helminth infection

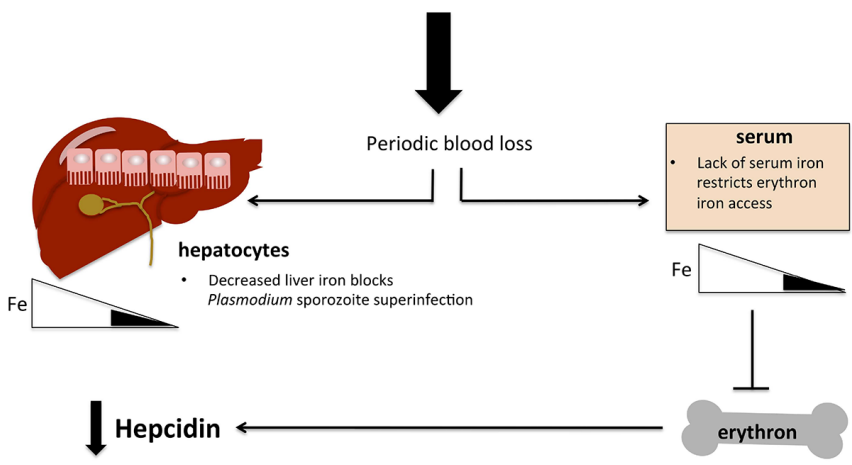

FIGURE 1 | Hypotheses for the effect of co-infections on iron

metabolism. (A) Possible implications of blood-stage malaria infection on host susceptibility to other infections. Blood-stage parasitemia causes hepcidin upregulation, which in turn routes iron away from hepatocytes and lowers serum iron levels, blocking the erythron's access to iron and causing anemia. Lowered hepatocyte iron levels prevent the establishment of a second malaria infection (superinfection) by blocking liver-stage growth. Hepcidin also causes iron levels to increase in macrophages and potentially in enterocytes as well, thus possibly giving an advantage to pathogens that exploit the macrophage niche (non-typhoidal salmonella, tuberculosis, Toxoplasma) and those that require iron in enterocytes (Toxoplasma).

(B) Hypothesized effect of HCV infection on malaria susceptibility. Long-term HCV infection causes hepcidin suppression, thus increasing liver iron stores and plausibly increasing malaria susceptibility. (C) Potential impact of intestinal helminth infection. Helminths cause periodic blood loss, which restricts iron to the liver, potentially blocking malaria liver-stage infection as in (A). Decreases in serum restrict iron availability for erythropoiesis, causing anemia, which in turn causes the downregulation of hepcidin. 
higher HIV viral load (Kublin et al., 2005). In turn, HIV-infected individuals are at greater risk of malarial infection (Patnaik et al., 2005). HIV affects iron metabolism in a complex manner: HIV is commonly associated with anemia, and in one study, hepcidin levels were shown to be elevated in HIV infection and inversely correlated with CD4 counts (Wisaksana et al., 2013). Conversely, increased iron stores in HIV-infected individuals are associated with mortality (McDermid et al., 2007, 2009) and risk of tuberculosis infection (McDermid et al., 2013). In an HIV-infected host, how do perturbations in iron and hepcidin modulate host susceptibility to malaria? Interestingly, HIV antiviral drugs have been found to demonstrate anti-Plasmodium activity at asexual (Skinner-Adams et al., 2004), liver (Hobbs et al., 2009), and gametocyte (Hobbs et al., 2013) life stages. However the effect of combination therapy on Plasmodium in HIV-1positive patients, and the interactions of anti-retrovirals with antimalarials and their overall effect on iron metabolism are difficult to predict.

Chronic hepatitis $\mathrm{C}$ virus (HCV) infection leads to hepcidin suppression and concomitant hepatic iron overload (Girelli et al., 2009). Might this increased iron lead to increased malaria susceptibility? Exposure of mice to HCV RNA induces a type 1 interferon response that has the effect of reducing parasite liver infection 48 h later (Liehl et al., 2014), but a long-term productive HCV infection and consequent iron overload may affect malaria risk very differently. More studies are needed to establish how chronic viral infections alter iron metabolism and malaria susceptibility.

Another unresolved issue is the effects of malaria on toxoplasmosis susceptibility. Toxoplasma gondii is carried asymptomatically by a large proportion of the human population, and it can cause life-threatening infections in pregnant women and the immunosuppressed. Toxoplasma infects hosts via enterocytes and can live in host macrophages (Hunter and Sibley, 2012). One study has shown that interferon gamma causes the death of Toxoplasma in enterocytes in a manner dependent on the depletion of intracellular iron stores (Dimier and Bout, 1998). Upregulation of hepcidin by a malaria infection would likely route iron toward enterocytes and macrophages, thus plausibly increasing host susceptibility and/or tolerance of Toxoplasma. If Toxoplasma does require intracellular iron stores to withstand the host response, then hepcidin upregulation and subsequent routing of iron to macrophages and enterocytes might increase host susceptibility to Toxoplasma infection.

A major cause of iron deficiency and anemia in the developing world is intestinal helminth infection. Infection with helminths is strongly associated with anemia in children (Calis et al., 2008; Jonker et al., 2012a); and de-worming treatment at the population level decreases anemia levels (reviewed in Smith and Brooker, 2010). Complex data exist on the contributions of intestinal helminth infections to malaria susceptibility and severity (Salgame et al., 2013). Early studies claimed that high-population helminth infection was associated with a striking lack of malaria, which the authors suggested was due to the nutritional perturbations associated with the worms (Murray et al., 1977). This finding was corroborated by more recent evidence suggesting that helminth infection in young children may be associated with fewer and later malaria episodes (Lyke et al., 2005), or protection from some of the potentially severe manifestations of malaria infections (Nacher et al., 2001, 2002). These effects remain controversial; further work has shown associations between helminths and higher parasitemia (Degarege et al., 2012) or severe malaria (Le Hesran et al., 2004). A single study found that infection with filarial worms ameliorated the drop in hemoglobin and reduced inflammatory cytokine production during malaria infection (Dolo et al., 2012). The impact of helminth infections on hepcidin is not well established, but it is plausible that helminth infection may, through periodic intestinal bleeding, cause constitutionally low hepcidin, as the body attempts to recoup the lost iron. At the same time, by causing iron deficiency, helminth infection may decrease host susceptibility to malaria or accentuate the effects of a hepcidin increase in infection.

Most laboratory studies on iron/infection interactions to date have focused on the effect of a single pathogen. Throughout human history, however, as well as in developing nations where infectious disease is still highly prevalent, susceptible individuals are frequently prey to multiple pathogens simultaneously. Understanding how pathogens affect iron metabolism and thereby modulate host susceptibility to other infections is likely to prove important both for its implications on treatment and on public health recommendations.

\section{SUPPRESSION OF HEPCIDIN IN SEVERE MALARIA SYNDROMES}

Although the majority of studies on hepcidin and malaria have demonstrated an upregulation of hepcidin in malaria infection, three recent studies have shown that in certain circumstances, hepcidin suppression may also occur. One study found that among all children presenting with malaria, those with severe anemia had the lowest hepcidin levels (Casals-Pascual et al., 2012). A further study (Burte et al., 2013) demonstrated that children with uncomplicated malaria had higher hepcidin levels than those who could be classified as either presenting with severe anemia (in all studies cited, severe anemia was defined as $\mathrm{Hb} \leq 5 \mathrm{~g} / \mathrm{L}$ ) or cerebral malaria. Finally, a group of children with severe malarial anemia exhibited very low hepcidin serum levels (over $50 \%$ were undetectable by the study's method; Jonker et al., 2013).

Taken together, these studies clearly indicate that in severe malarial anemia, the signaling pathway that suppresses hepcidin can override the activation pathways associated with parasitemia. The mechanisms of hepcidin suppression by erythropoietic drive, hypoxia, or iron deficiency have not yet been well defined, but some groups have posited the existence of a bone marrow-secreted factor that suppresses hepcidin during erythropoiesis (Ginzburg and Rivella, 2011). In the two human studies that compared erythropoietin levels with serum hepcidin in malaria infection, erythropoietin and hepcidin were negatively associated (CasalsPascual et al., 2012; Jonker et al., 2013). One animal study has also demonstrated a significant negative correlation between serum erythropoietin and hepcidin liver message in mice infected with $P$. berghei (Wang et al., 2011).

Further studies utilizing animal models of severe malarial anemia will likely be required to explore this aspect of iron control 
in malaria infection. In addition, the role of iron and hepcidin in cerebral malaria requires investigation.

\section{MALARIA PREVENTION EFFORTS REDUCE ANEMIA PREVALENCE}

A recently published meta-analysis of global anemia prevalence estimated that in 2010, the prevalence of anemia was $32.9 \%$, accounting for $8.8 \%$ of total years of life lived with disability worldwide (Kassebaum et al., 2014). In malaria-endemic countries, malaria is a major contributor to anemia at the population level: the authors estimated that in sub-Saharan African, $24.7 \%$ of anemia is attributable to malaria (Kassebaum et al., 2014). Therefore, interventions that reduce the prevalence of malaria could be expected to result in a reduction in the severity and prevalence of anemia.

The global community is currently using multiple complementary tools to reduce malaria at the population level. Intermittent preventative treatment (IPT) refers to the practice of administering antimalarial drugs presumptively during the especially vulnerable periods of pregnancy or early childhood. Insecticidetreated bed nets (ITNs) reduce nocturnal bites from Anopheles mosquitoes (Hill et al., 2006). Both interventions serve to protect the recipient and to reduce transmission at a population level (Greenwood, 2004).

A recent Cochrane review examining the effects of IPT for children under 5 concluded that of five West African trials examined, there was likely a significant reduction overall in moderate anemia incidence in areas with seasonal $P$. falciparum malaria transmission (Meremikwu et al., 2012). Of the two trials, which considered severe anemia as a separate outcome, both also found a significant reduction as a result of IPT (Dicko et al., 2011; Konate et al., 2011). This is consistent with the findings of a previous Cochrane review, which had also concluded that IPT in pregnant women was similarly associated with decreased rates of both severe anemia and all anemia (Garner and Gulmezoglu, 2006).

Fewer studies have examined the effects of the introduction of ITNs only on anemia prevalence, but earlier introduction of ITNs is associated with a statistically significant reduction in anemia prevalence in children at 6-12 months of age (Muller et al., 2006). In another study, hemoglobin levels were found to be significantly associated with the use of ITNs in young children (Holtz et al., 2002).

Why does reducing malaria infection have such a profound effect on population anemia levels? The relationship between malaria control and anemia risk may be partially dependent on hepcidin and its effects on iron absorption and utilization (as well as by reducing the well-known inhibitory effects of malaria on erythropoiesis). The hepcidin increases associated with malaria infection prevent efficient iron uptake. Iron supplementation has been shown to be less effective in areas with high malaria transmission (Gera et al., 2007).

Two studies have delineated the effects of malaria infection and hepcidin increase on the incorporation of orally administered iron into erythrocytes (Doherty et al., 2008; Cercamondi et al., 2010). In a study of young Beninese women, asymptomatic parasitemia was associated with poor incorporation of orally administered iron into RBCs, an effect that did not extend to parentally administered iron. Treatment and resolution of parasitemia resulted in a decrease in serum hepcidin and improved absorption of oral iron supplements (Cercamondi et al., 2010).

Similarly, young children with post-malarial anemia were shown to poorly absorb orally administered iron supplements (Doherty et al., 2008). Hepcidin levels in sera of these children were measured subsequently; these hepcidin levels were shown to be the best predictors of iron absorption (Prentice et al., 2012). Furthermore, despite poor oral absorption of iron, these children showed a more rapid hematological recovery than anemic and aparasitemic children, suggesting that hepcidin upregulation in malaria may contribute to relocalization of iron to macrophages, rather than true iron deficiency.

Populations with a high prevalence of malaria infections may therefore suffer both from anemia as a direct consequence of malaria infections, but also from poor utilization of oral iron as a result of chronically upregulated hepcidin.

\section{DISCUSSION AND RECOMMENDATIONS}

Anemia continues to be one of the most common causes of disability worldwide; while Plasmodium is one of the most prevalent human pathogens. The relationship between the two is complex: low iron status may protect against malaria infection, but malaria infection in turn is linked with anemia at both the individual and population levels. New clues to this relationship have been obtained through our improved understanding of iron metabolism.

Specifically, the relatively recent identification of the human iron hormone hepcidin has allowed us to begin to explore these relationships in more depth. Hepcidin is upregulated in malaria infection, likely contributing to anemia through the relocalization of iron to macrophages and the prevention of iron uptake from the diet. However, in certain severe malaria syndromes, hepcidin may be suppressed. The mechanisms behind both hepcidin's increase and its suppression in malaria are currently unclear.

At the moment, action on three fronts is required. First, we must implement our improved understanding of the ironmalaria relationship toward optimizing malaria prevention and anemia treatment. Research findings support more nuanced iron supplementation regimens, rather than the blanket approach of supplementing, or not supplementing, whole populations. Supplementation could be scheduled around the malaria season (Atkinson et al., 2014), routed to the most iron-deprived children or pregnant women, or targeted based on hepcidin levels as a proposed biomarker (Pasricha et al., 2014). At the very least, the findings of the last decade of research since the Pemba trial argues very strongly that iron supplementation should be carried out in concert with malaria control efforts; and that improved malaria control may itself be effective as a means of decreasing anemia at the population level.

Second, clinically focused research is required to develop better and rationally informed therapeutics for those who become infected. For example, understanding the mechanisms underlying hepcidin upregulation in malaria could be the first step toward the development of drugs that, given concurrently with antimalarial treatment, repress hepcidin and speed recovery from 
anemia. Carefully designed in vivo and in vitro studies could explore the role that hepcidin may play in mediating the outcomes of co-infections between malaria and other protozoan, bacterial, or viral pathogens, providing vital information for treating individuals and populations exposed to multiple pathogens simultaneously.

Finally, in the longer term, basic research should focus on an improved understanding of the host-pathogen tug-of-war over iron metabolism. How does malaria acquire iron at a molecular level in its many life stages? Do malaria pathogens show different phenotypes in iron-deprived hosts? Armed with new tools to explore pathogen biology and iron metabolism, we have a chance to answer questions of immediate clinical importance, and to advance the basic science behind our understanding of the host-parasite relationship.

\section{ACKNOWLEDGMENTS}

Natasha Spottiswoode and Patrick E. Duffy are supported by the Intramural Research Program of NIAID, NIH. Natasha Spottiswoode was also supported through the NIH Oxford - Cambridge Scholars Program during the period of writing. Hal Drakesmith is supported by the Medical Research Council, UK and by the Bill and Melinda Gates Foundation.

\section{REFERENCES}

Abboud, S., and Haile, D. J. (2000). A novel mammalian iron-regulated protein involved in intracellular iron metabolism. J. Biol. Chem. 275, 19906-19912. doi: 10.1074/jbc.M000713200

Armitage, A. E., Eddowes, L. A., Gileadi, U., Cole, S., Spottiswoode, N., Selvakumar T. A., et al. (2011). Hepcidin regulation by innate immune and infectious stimuli. Blood 118, 4129-4139. doi: 10.1182/blood-2011-04-351957

Armitage, A. E., Pinches, R., Eddowes, L. A., Newbold, C. I., and Drakesmith, H. (2009). Plasmodium falciparum infected erythrocytes induce hepcidin (HAMP) mRNA synthesis by peripheral blood mononuclear cells. Br. J. Haematol. 147, 769-771. doi: 10.1111/j.1365-2141.2009.07880.x

Atkinson, S. H., Armitage, A. E., Khandwala, S., Mwangi, T. W., Uyoga, S., Bejon, P. A., etal. (2014). Combinatorial effects of malaria season, iron deficiency and inflammation determine plasma hepcidin concentration in African children. Blood doi: 10.1182/blood-2013-10533000 [Epub ahead of print].

Besson-Fournier, C., Latour, C., Kautz, L., Bertrand, J., Ganz, T., Roth, M. P., et al. (2012). Induction of activin B by inflammatory stimuli up-regulates expression of the iron-regulatory peptide hepcidin through Smad1/5/8 signaling. Blood 120 , 431-439. doi: 10.1182/blood-2012-02-411470

Bridle, K. R., Frazer, D. M., Wilkins, S. J., Dixon, J. L., Purdie, D. M., Crawford, D. H., et al. (2003). Disrupted hepcidin regulation in HFE-associated haemochromatosis and the liver as a regulator of body iron homoeostasis. Lancet 361, 669-673. doi: 10.1016/S0140-6736(03)12602-5

Burte, F., Brown, B. J., Orimadegun, A. E., Ajetunmobi, W. A., Afolabi, N. K., Akinkunmi, F., et al. (2013). Circulatory hepcidin is associated with the antiinflammatory response but not with iron or anemic status in childhood malaria. Blood 121, 3016-3022. doi: 10.1182/blood-2012-10-461418

Calis, J. C., Phiri, K. S., Faragher, E. B., Brabin, B. J., Bates, I., Cuevas, L. E., et al. (2008). Severe anemia in Malawian children. N. Engl. J. Med. 358, 888-899. doi 10.1056/NEJMoa072727

Casals-Pascual, C., Huang, H., Lakhal-Littleton, S., Thezenas, M. L., Kai, O., Newton C. R., et al. (2012). Hepcidin demonstrates a biphasic association with anemia in acute Plasmodium falciparum malaria. Haematologica 97, 1695-1698. doi: 10.3324/haematol.2012.065854

Cercamondi, C. I., Egli, I. M., Ahouandjinou, E., Dossa, R., Zeder, C., Salami, L., et al. (2010). Afebrile Plasmodium falciparum parasitemia decreases absorption of fortification iron but does not affect systemic iron utilization: a double stableisotope study in young Beninese women. Am. J. Clin. Nutr. 92, 1385-1392. doi: 10.3945/ajen.2010.30051
Cunnington, A. J., de Souza, J. B., Walther, M., and Riley, E. M. (2012) Malaria impairs resistance to Salmonella through heme- and heme oxygenasedependent dysfunctional granulocyte mobilization. Nat. Med. 18, 120-127. doi: $10.1038 / \mathrm{nm} .2601$

de Mast, Q., Nadjm, B., Reyburn, H., Kemna, E. H., Amos, B., Laarakkers, C. M., et al. (2009a). Assessment of urinary concentrations of hepcidin provides novel insight into disturbances in iron homeostasis during malarial infection. J. Infect. Dis. 199, 253-262. doi: 10.1086/595790

de Mast, Q., van Dongen-Lases, E. C., Swinkels, D. W., Nieman, A. E., Roestenberg, M., Druilhe, P., et al. (2009b). Mild increases in serum hepcidin and interleukin-6 concentrations impair iron incorporation in haemoglobin during an experimental human malaria infection. Br. J. Haematol. 145, 657-664. doi: 10.1111/j.1365-2141.2009.07664.x

de Mast, Q., Syafruddin, D., Keijmel, S., Riekerink, T. O., Deky, O., Asih, P. B., et al. (2010). Increased serum hepcidin and alterations in blood iron parameters associated with asymptomatic $P$. falciparum and $P$. vivax malaria. Haematologica 95, 1068-1074. doi: 10.3324/haematol.2009.019331

Degarege, A., Legesse, M., Medhin, G., Animut, A., and Erko, B. (2012). Malaria and related outcomes in patients with intestinal helminths: a cross-sectional study. BMC Infect. Dis. 12:291. doi: 10.1186/1471-2334-12-291

Desai, M. R., Mei, J. V., Kariuki, S. K., Wannemuehler, K. A., Phillips-Howard, P. A., Nahlen, B. L., et al. (2003). Randomized, controlled trial of daily iron supplementation and intermittent sulfadoxine-pyrimethamine for the treatment of mild childhood anemia in western Kenya. J. Infect. Dis. 187, 658-666. doi: $10.1086 / 367986$

Dicko, A., Diallo, A. I., Tembine, I., Dicko, Y., Dara, N., Sidibe, Y., et al. (2011). Intermittent preventive treatment of malaria provides substantial protection against malaria in children already protected by an insecticide-treated bednet in Mali: a randomised, double-blind, placebo-controlled trial. PLoS Med. 8:e1000407. doi: 10.1371/journal.pmed.1000407

Dimier, I. H., and Bout, D. T. (1998). Interferon-gamma-activated primary enterocytes inhibit Toxoplasma gondii replication: a role for intracellular iron. Immunology 94, 488-495. doi: 10.1046/j.1365-2567.1998. 00553.x

Doherty, C. P., Cox, S. E., Fulford, A. J., Austin, S., Hilmers, D. C., Abrams, S. A., et al. (2008). Iron incorporation and post-malaria anaemia. PLoS ONE 3:e2133. doi: 10.1371/journal.pone.0002133

Dolo, H., Coulibaly, Y. I., Dembele, B., Konate, S., Coulibaly, S. Y., Doumbia, S. S., et al. (2012). Filariasis attenuates anemia and proinflammatory responses associated with clinical malaria: a matched prospective study in children and young adults. PLoS Negl. Trop. Dis. 6:e1890. doi: 10.1371/journal.pntd. 0001890

Donovan, A., Brownlie, A., Zhou, Y., Shepard, J., Pratt, S. J., Moynihan, J., etal. (2000). Positional cloning of zebrafish ferroportinl identifies a conserved vertebrate iron exporter. Nature 403, 776-781. doi: 10.1038/350 01596

Drakesmith, H., Schimanski, L. M., Ormerod, E., Merryweather-Clarke, A. T., Viprakasit, V., Edwards, J. P., et al. (2005). Resistance to hepcidin is conferred by hemochromatosis-associated mutations of ferroportin. Blood 106, 1092-1097. doi: 10.1182/blood-2005-02-0561

Ferrer, P., Tripathi, A. K., Clark, M. A., Hand, C. C., Rienhoff, H. Y. Jr., and Sullivan, D. J. Jr. (2012). Antimalarial iron chelator, FBS0701, shows asexual and gametocyte Plasmodium falciparum activity and single oral dose cure in a murine malaria model. PLoS ONE 7:e37171. doi: 10.1371/journal.pone. 0037171

Finberg, K. E., Heeney, M. M., Campagna, D. R., Aydinok, Y., Pearson, H. A., Hartman, K. R., et al. (2008). Mutations in TMPRSS6 cause iron-refractory iron deficiency anemia (IRIDA). Nat. Genet. 40, 569-571. doi: 10.1038/ ng. 130

Friedman, J. F., Kurtis, J. D., Kabyemela, E. R., Fried, M., and Duffy, P. E. (2009). The iron trap: iron, malaria and anemia at the mother-child interface. Microbes Infect. 11, 460-466. doi: 10.1016/j.micinf.2009.02.006

Fritsch, G., Treumer, J., Spira, D. T., and Jung, A. (1985). Plasmodium vinckei: suppression of mouse infections with desferrioxamine B. Exp. Parasitol. 60, 171174. doi: 10.1016/0014-4894(85)90020-7

Garner, P., and Gulmezoglu, A. M. (2006). Drugs for preventing malaria in pregnant women. Cochrane Database Syst. Rev. CD000169. doi: 10.1002/14651858.CD000169.pub2 
Gera, T., Sachdev, H. P., Nestel, P., and Sachdev, S. S. (2007). Effect of iron supplementation on haemoglobin response in children: systematic review of randomised controlled trials. J. Pediatr. Gastroenterol. Nutr. 44, 468-486. doi: 10.1097/01.mpg.0000243440.85452.38

Ginzburg, Y., and Rivella, S. (2011). Beta-thalassemia: a model for elucidating the dynamic regulation of ineffective erythropoiesis and iron metabolism. Blood 118 4321-4330. doi: 10.1182/blood-2011-03-283614

Girelli, D., Pasino, M., Goodnough, J. B., Nemeth, E., Guido, M., Castagna, A., et al. (2009). Reduced serum hepcidin levels in patients with chronic hepatitis C. J. Hepatol. 51, 845-852. doi: 10.1016/j.jhep.2009.06.027

Greenwood, B. (2004). The use of anti-malarial drugs to prevent malaria in the population of malaria-endemic areas. Am. J. Trop. Med. Hyg 70, 1-7.

Gwamaka, M., Kurtis, J. D., Sorensen, B. E., Holte, S., Morrison, R., Mutabingwa T. K., et al. (2012). Iron deficiency protects against severe Plasmodium falciparum malaria and death in young children. Clin. Infect. Dis. 54, 1137-1144. doi: $10.1093 / \mathrm{cid} / \mathrm{cis} 010$

Hershko, C., and Peto, T. E. (1988). Deferoxamine inhibition of malaria is independent of host iron status. J. Exp. Med. 168, 375-387. doi: 10.1084/jem.168.1.375

Hill, J., Lines, J., and Rowland, M. (2006). Insecticide-treated nets. Adv. Parasitol. 61, 77-128. doi: 10.1016/S0065-308X(05)61003-2

Hobbs, C. V., Tanaka, T. Q., Muratova, O., Van Vliet, J., Borkowsky, W., Williamson, K. C., et al. (2013). HIV treatments have malaria gametocyte killing and transmission blocking activity. J. Infect. Dis. 208, 139-148. doi: 10.1093/infdis/jit132

Hobbs, C. V., Voza, T., Coppi, A., Kirmse, B., Marsh, K., Borkowsky, W., et al. (2009). HIV protease inhibitors inhibit the development of preerythrocytic-stage plasmodium parasites. J. Infect. Dis. 199, 134-141. doi: 10.1086/594369

Holtz, T. H., Marum, L. H., Mkandala, C., Chizani, N., Roberts, J. M., Macheso, A., et al. (2002). Insecticide-treated bednet use, anaemia, and malaria parasitaemia in Blantyre District, Malawi. Trop. Med. Int. Health 7, 220-230. doi: 10.1046/j.13653156.2002.00846.x

Howard, C. T., McKakpo, U. S., Quakyi, I. A., Bosompem, K. M., Addison, E. A., Sun, K., et al. (2007). Relationship of hepcidin with parasitemia and anemia among patients with uncomplicated Plasmodium falciparum malaria in Ghana. Am. J. Trop. Med. Hyg. 77, 623-626.

Hunter, C. A., and Sibley, L. D. (2012). Modulation of innate immunity by Toxoplasma gondii virulence effectors. Nat. Rev. Microbiol. 10, 766-778. doi: 10.1038/nrmicro2858

Jonker, F. A., Calis, J. C., Phiri, K., Brienen, E. A., Khoffi, H., Brabin, B. J., et al. (2012a). Real-time PCR demonstrates Ancylostoma duodenale is a key factor in the etiology of severe anemia and iron deficiency in Malawian preschool children. PLoS Negl. Trop. Dis. 6:e1555. doi: 10.1371/journal.pntd.00 01555

Jonker, F. A., Calis, J. C., van Hensbroek, M. B., Phiri, K., Geskus, R. B., Brabin, B. J., et al. (2012b). Iron status predicts malaria risk in Malawian preschool children. PLoS ONE 7:e42670. doi: 10.1371/journal.pone.0042670

Jonker, F. A., Calis, J. C., Phiri, K., Kraaijenhagen, R. J., Brabin, B. J., Faragher, B., et al. (2013). Low hepcidin levels in severely anemic malawian children with high incidence of infectious diseases and bone marrow iron deficiency. PLoS ONE 8:e78964. doi: 10.1371/journal.pone.0078964

Kabyemela, E. R., Fried, M., Kurtis, J. D., Mutabingwa, T. K., and Duffy, P. E. (2008). Decreased susceptibility to Plasmodium falciparum infection in pregnant women with iron deficiency. J. Infect. Dis. 198, 163-166. doi: 10.1086/ 589512

Kassebaum, N. J., Jasrasaria, R., Naghavi, M., Wulf, S. K., Johns, N., Lozano, R., et al. (2014). A systematic analysis of global anemia burden from 1990 to 2010. Blood 123, 615-624. doi: 10.1182/blood-2013-06-508325

Konate, A. T., Yaro, J. B., Ouedraogo, A. Z., Diarra, A., Gansane, A., Soulama, I., et al. (2011). Intermittent preventive treatment of malaria provides substantial protection against malaria in children already protected by an insecticide-treated bednet in Burkina Faso: a randomised, double-blind, placebo-controlled trial. PLoS Med. 8:e1000408. doi: 10.1371/journal.pmed.10 00408

Krause, A., Neitz, S., Magert, H. J., Schulz, A., Forssmann, W. G., SchulzKnappe, P., et al. (2000). LEAP-1, a novel highly disulfide-bonded human peptide, exhibits antimicrobial activity. FEBS Lett. 480, 147-150. doi: 10.1016/S00145793(00)01920-7
Kublin, J. G., Patnaik, P., Jere, C. S., Miller, W. C., Hoffman, I. F., Chimbiya, N., et al. (2005). Effect of Plasmodium falciparum malaria on concentration of HIV1-RNA in the blood of adults in rural Malawi: a prospective cohort study. Lancet 365, 233-240.

Le Hesran, J. Y., Akiana, J., Ndiaye el, H. M., Dia, M., Senghor, P., and Konate, L. (2004). Severe malaria attack is associated with high prevalence of Ascaris lumbricoides infection among children in rural Senegal. Trans. Roy. Soc. Trop. Med. Hyg. 98, 397-399. doi: 10.1016/j.trstmh.2003. 10.009

Liehl, P., Zuzarte-Luis, V., Chan, J., Zillinger, T., Baptista, F., Carapau, D., etal. (2014). Host-cell sensors for Plasmodium activate innate immunity against liver-stage infection. Nat. Med. 20, 47-53. doi: 10.1038/ nm. 3424

Lyke, K. E., Dicko, A., Dabo, A., Sangare, L., Kone, A., Coulibaly, D., et al. (2005). Association of Schistosoma haematobium infection with protection against acute Plasmodium falciparum malaria in Malian children. Am. J. Trop. Med. Hyg. 73, 1124-1130.

Mabey, D. C., Brown, A., and Greenwood, B. M. (1987). Plasmodium falciparum malaria and Salmonella infections in Gambian children. J. Infect. Dis. 155, 13191321. doi: 10.1093/infdis/155.6.1319

Mabeza, G. F., Loyevsky, M., Gordeuk, V. R., and Weiss, G. (1999). Iron chelation therapy for malaria: a review. Pharmacol. Ther. 81, 53-75. doi: 10.1016/S01637258(98)00037-0

Manning, L., Laman, M., Rosanas-Urgell, A., Michon, P., Aipit, S., Bona, C., et al. (2012). Severe anemia in Papua New Guinean children from a malariaendemic area: a case-control etiologic study. PLoS Negl. Trop. Dis. 6:e1972. doi: 10.1371/journal.pntd.0001972

Matsuzaki-Moriya, C., Tu, L., Ishida, H., Imai, T., Suzue, K., Hirai, M., etal. (2011). A critical role for phagocytosis in resistance to malaria in iron-deficient mice. Eur. J. Immunol. 41, 1365-1375. doi: 10.1002/eji.201 040942

McDermid, J. M., Hennig, B. J., van der Sande, M., Hill, A. V., Whittle, H. C., Jaye, A., et al. (2013). Host iron redistribution as a risk factor for incident tuberculosis in HIV infection: an 11-year retrospective cohort study. BMC Infect. Dis. 13:48. doi: 10.1186/1471-2334-13-48

McDermid, J. M., Jaye, A., Schim van der Loeff, M. F., Todd, J., Bates, C., Austin, S., et al. (2007). Elevated iron status strongly predicts mortality in West African adults with HIV infection. J. Acquir. Immune Defic. Syndr. 46, 498-507. doi: 10.1097/QAI.0b013e31815b2d4b

McDermid, J. M., and Prentice, A. M. (2006). Iron and infection: effects of host iron status and the iron-regulatory genes haptoglobin and NRAMP1 (SLC11A1) on host-pathogen interactions in tuberculosis and HIV. Clin. Sci. (Lond.) 110, 503-524. doi: 10.1042/CS20050273

McDermid, J. M., van der Loeff, M. F., Jaye, A., Hennig, B. J., Bates, C., Todd, J., et al. (2009). Mortality in HIV infection is independently predicted by host iron status and SLC11A1 and HP genotypes, with new evidence of a genenutrient interaction. Am. J. Clin. Nutr. 90, 225-233. doi: 10.3945/ajcn.2009. 27709

McKie, A. T., Marciani, P., Rolfs, A., Brennan, K., Wehr, K., Barrow, D., et al. (2000). A novel duodenal iron-regulated transporter, IREG1, implicated in the basolateral transfer of iron to the circulation. Mol. Cell 5, 299-309. doi: 10.1016/S10972765(00)80425-6

Menendez, C., Todd, J., Alonso, P. L., Francis, N., Lulat, S., Ceesay, S., et al. (1994). The effects of iron supplementation during pregnancy, given by traditional birth attendants, on the prevalence of anaemia and malaria. Trans. Roy. Soc. Trop. Med. Hyg. 88, 590-593. doi: 10.1016/0035-9203(94) 90176-7

Meremikwu, M. M., Donegan, S., Sinclair, D., Esu, E., and Oringanje, C. (2012). Intermittent preventive treatment for malaria in children living in areas with seasonal transmission. Cochrane Database Syst. Rev. 2, CD003756. doi: 10.1002/14651858.CD003756.pub4

Muller, O., Traore, C., Kouyate, B., Ye, Y., Frey, C., Coulibaly, B., et al. (2006). Effects of insecticide-treated bednets during early infancy in an African area of intense malaria transmission: a randomized controlled trial. Bull. World Health Organ. 84, 120-126. doi: 10.2471/BLT.05.023150

Murray, M. J., and Murray, A. B. (1977). Starvation suppression and refeeding activation of infection. An ecological necessity? Lancet 1, 123-125. doi: 10.1016/S0140-6736(77)91710-X 
Murray, M. J., Murray, A. B., and Murray, C. J. (1980). An ecological interdependence of diet and disease? A study of infection in one tribe consuming two different diets. Am. J. Clin. Nutr. 33, 697-701.

Murray, M. J., Murray, A. B., Murray, M. B., and Murray, C. J. (1977). Parotid enlargement, forehead edema, and suppression of malaria as nutritional consequences of ascariasis. Am. J. Clin. Nutr. 30, 2117-2121.

Murray, M. J., Murray, A. B., Murray, M. B., and Murray, C. J. (1978). The adverse effect of iron repletion on the course of certain infections. Br. Med. J. 2, 1113 1115. doi: 10.1136/bmj.2.6145.1113

Murray, M. J., Murray, N. J., Murray, A. B., and Murray, M. B. (1975). Refeeding-malaria and hyperferraemia. Lancet 1, 653-654. doi: 10.1016/S01406736(75)91758-4

Nacher, M., McGready, R., Stepniewska, K., Cho, T., Looareesuwan, S., White, N. J., et al. (2003). Haematinic treatment of anaemia increases the risk of Plasmodium vivax malaria in pregnancy. Trans. Roy. Soc. Trop. Med. Hyg. 97, 273-276. doi: 10.1016/S0035-9203(03)90140-4

Nacher, M., Singhasivanon, P., Silachamroon, U., Treeprasertsuk, S., Vannaphan, S., Traore, B., et al. (2001). Helminth infections are associated with protection from malaria-related acute renal failure and jaundice in Thailand. Am. J. Trop. Med. Hyg. 65, 834-836.

Nacher, M., Singhasivanon, P., Traore, B., Vannaphan, S., Gay, F., Chindanond, D., et al. (2002). Helminth infections are associated with protection from cerebral malaria and increased nitrogen derivatives concentrations in Thailand. Am. J. Trop. Med. Hyg. 66, 304-309.

Nemeth, E., Tuttle, M. S., Powelson, J., Vaughn, M. B., Donovan, A., Ward, D. M., et al. (2004). Hepcidin regulates cellular iron efflux by binding to ferroportin and inducing its internalization. Science 306, 2090-2093. doi: 10.1126/science. 1104742

Nemeth, E., Valore, E. V., Territo, M., Schiller, G., Lichtenstein, A., and Ganz, T. (2003). Hepcidin, a putative mediator of anemia of inflammation, is a type II acute-phase protein. Blood 101, 2461-2463. doi: 10.1182/blood-200210-3235

Nicolas, G., Bennoun, M., Devaux, I., Beaumont, C., Grandchamp, B., Kahn, A., et al. (2001). Lack of hepcidin gene expression and severe tissue iron overload in upstream stimulatory factor 2 (USF2) knockout mice. Proc. Natl. Acad. Sci. U.S.A. 98, 8780-8785. doi: 10.1073/pnas.151179498

Nicolas, G., Bennoun, M., Porteu, A., Mativet, S., Beaumont, C., Grandchamp, B., etal. (2002a). Severe iron deficiency anemia in transgenic mice expressing liver hepcidin. Proc. Natl. Acad. Sci. U.S.A. 99, 4596-4601. doi: 10.1073/pnas.072632499

Nicolas, G., Chauvet, C., Viatte, L., Danan, J. L., Bigard, X., Devaux, I., et al. (2002b). The gene encoding the iron regulatory peptide hepcidin is regulated by anemia, hypoxia, and inflammation. J. Clin. Invest. 110, 1037-1044. doi: 10.1172/JCI0215686

Nyakeriga, A. M., Troye-Blomberg, M., Dorfman, J. R., Alexander, N. D., Back, R., Kortok, M., et al. (2004). Iron deficiency and malaria among children living on the coast of Kenya. J. Infect. Dis. 190, 439-447. doi: 10.1086/ 422331

Ojukwu, J. U., Okebe, J. U., Yahav, D., and Paul, M. (2009). Oral iron supplementation for preventing or treating anaemia among children in malaria-endemic areas. Cochrane Database Syst. Rev. CD006589. doi: 10.1002/14651858.CD0065 89.pub2

Okebe, J. U., Yahav, D., Shbita, R., and Paul, M. (2011). Oral iron supplements for children in malaria-endemic areas. Cochrane Database Syst. Rev. CD006589. doi: 10.1002/14651858.CD006589.pub3

Oppenheimer, S. J., Macfarlane, S. B., Moody, J. B., and Harrison, C. (1986). Total dose iron infusion, malaria and pregnancy in Papua New Guinea. Trans. Roy. Soc. Trop. Med. Hyg. 80, 818-822. doi: 10.1016/0035-9203(86)90393-7

Ouedraogo, H. Z., Dramaix-Wilmet, M., Zeba, A. N., Hennart, P., and Donnen, P. (2008). Effect of iron or multiple micronutrient supplements on the prevalence of anaemia among anaemic young children of a malaria-endemic area: a randomized double-blind trial. Trop. Med. Intl. Health 13, 1257-1266. doi: 10.1111/j.13653156.2008.02138.x

Park, C. H., Valore, E. V., Waring, A. J., and Ganz, T. (2001). Hepcidin, a urinary antimicrobial peptide synthesized in the liver. J. Biol. Chem. 276, 7806-7810. doi: 10.1074/jbc.M008922200

Pasricha, S. R., Atkinson, S. H., Armitage, A. E., Khandwala, S., Veenemans, J., Cox, S. E., et al. (2014). Expression of the iron hormone hepcidin distinguishes different types of anemia in african children. Sci. Transl. Med. 6, 235re233. doi: 10.1126/scitranslmed.3008249

Patnaik, P., Jere, C. S., Miller, W. C., Hoffman, I. F., Wirima, J., Pendame, R., et al. (2005). Effects of HIV-1 serostatus, HIV-1 RNA concentration, and CD4 cell count on the incidence of malaria infection in a cohort of adults in rural Malawi. J. Infect. Dis. 192, 984-991. doi: 10.1086/432730

Pigeon, C., Ilyin, G., Courselaud, B., Leroyer, P., Turlin, B., Brissot, P., et al. (2001). A new mouse liver-specific gene, encoding a protein homologous to human antimicrobial peptide hepcidin, is overexpressed during iron overload. J. Biol. Chem. 276, 7811-7819. doi: 10.1074/jbc.M008923200

Pollack, S., Rossan, R. N., Davidson, D. E., and Escajadillo, A. (1987). Desferrioxamine suppresses Plasmodium falciparum in Aotus monkeys. Proc. Soc. Exp. Biol. Med. 184, 162-164. doi: 10.3181/00379727-184-42461

Portugal, S., Carret, C., Recker, M., Armitage, A. E., Goncalves, L. A., Epiphanio, S., et al. (2011). Host-mediated regulation of superinfection in malaria. Nat. Med. 17, 732-737. doi: 10.1038/nm.2368

Prentice, A. M., Doherty, C. P., Abrams, S. A., Cox, S. E., Atkinson, S. H., Verhoef, H., et al. (2012). Hepcidin is the major predictor of erythrocyte iron incorporation in anemic African children. Blood 119, 1922-1928. doi: 10.1182/blood-2011-11391219

Prentice, A. M., Verhoef, H., and Cerami, C. (2013). Iron fortification and malaria risk in children. JAMA 310, 914-915. doi: 10.1001/jama.20 13.6771

Raventos-Suarez, C., Pollack, S., and Nagel, R. L. (1982). Plasmodium falciparum: inhibition of in vitro growth by desferrioxamine. Am. J. Trop. Med. Hyg. 31, 919-922.

Rodriguez, M. H., and Jungery, M. (1986). A protein on Plasmodium falciparuminfected erythrocytes functions as a transferrin receptor. Nature 324, 388-391. doi: $10.1038 / 324388 \mathrm{a} 0$

Roetto, A., Papanikolaou, G., Politou, M., Alberti, F., Girelli, D., Christakis, J., et al. (2003). Mutant antimicrobial peptide hepcidin is associated with severe juvenile hemochromatosis. Nat. Genet. 33, 21-22. doi: 10.1038/ng1053

Salgame, P., Yap, G. S., and Gause, W. C. (2013). Effect of helminth-induced immunity on infections with microbial pathogens. Nat. Immunol. 14, 1118-1126. doi: 10.1038/ni.2736

Sazawal, S., Black, R. E., Ramsan, M., Chwaya, H. M., Stoltzfus, R. J., Dutta, A., et al. (2006). Effects of routine prophylactic supplementation with iron, and folic acid on admission to hospital, and mortality in preschool children in a high malaria transmission setting: community-based, randomised, placebo-controlled trial. Lancet 367, 133-143. doi: 10.1016/S0140-6736(06) 67962-2

Senga, E. L., Harper, G., Koshy, G., Kazembe, P. N., and Brabin, B. J. (2011). Reduced risk for placental malaria in iron deficient women. Malar. J. 10, 47. doi: 10.1186/1475-2875-10-47

Senga, E. L., Koshy, G., and Brabin, B. J. (2012). Zinc erythrocyte protoporphyrin as marker of malaria risk in pregnancy - a retrospective crosssectional and longitudinal study. Malar. J. 11, 249. doi: 10.1186/1475-287511-249

Skinner-Adams, T. S., McCarthy, J. S., Gardiner, D. L., Hilton, P. M., and Andrews, K. T. (2004). Antiretrovirals as antimalarial agents. J. Infect. Dis. 190, 1998-2000. doi: $10.1086 / 425584$

Smith, H. J., and Meremikwu, M. (2003). Iron chelating agents for treating malaria. Cochrane Database Syst. Rev. CD001474. doi: 10.1002/14651858.CD 001474

Smith, J. L., and Brooker, S. (2010). Impact of hookworm infection and deworming on anaemia in non-pregnant populations: a systematic review. Trop. Med. Int. Health 15, 776-795. doi: 10.1111/j.1365-3156.2010.02542.x

Thuma, P. E., Mabeza, G. F., Biemba, G., Bhat, G. J., McLaren, C. E., Moyo, V. M., et al. (1998). Effect of iron chelation therapy on mortality in Zambian children with cerebral malaria. Trans. Roy. Soc. Trop. Med. Hyg. 92, 214-218. doi: 10.1016/S0035-9203(98)90753-2

Traore, O., Carnevale, P., Kaptue-Noche, L., M’Bede, J., Desfontaine, M., Elion, J., et al. (1991). Preliminary report on the use of desferrioxamine in the treatment of Plasmodium falciparum malaria. Am. J. Hematol. 37, 206-208. doi: 10.1002/ajh.2830370316

van Santen, S., de Mast, Q., Swinkels, D. W., and van der Ven, A. J. (2013). The iron link between malaria and invasive non-typhoid Salmonella infections. Trends Parasitol. 29, 220-227. doi: 10.1016/j.pt.2013.03.006 
Wang, H. Z., He, Y. X., Yang, C. J., Zhou, W., and Zou, C. G. (2011). Hepcidin is regulated during blood-stage malaria and plays a protective role in malaria infection. J. Immunol. 187, 6410-6416. doi: 10.4049/jimmunol.11 01436

Wang, R. H., Li, C., Xu, X., Zheng, Y., Xiao, C., Zerfas, P., et al. (2005). A role of SMAD4 in iron metabolism through the positive regulation of hepcidin expression. Cell Metab. 2, 399-409. doi: 10.1016/j.cmet.2005. 10.010

Wisaksana, R., de Mast, Q., Alisjahbana, B., Jusuf, H., Sudjana, P., Indrati, A. R., et al. (2013). Inverse relationship of serum hepcidin levels with CD4 cell counts in HIV-infected patients selected from an Indonesian prospective cohort study. PLoS ONE 8:e79904. doi: 10.1371/journal.pone.0079904

Zlotkin, S., Newton, S., Aimone, A. M., Azindow, I., Amenga-Etego, S., Tchum, K., etal. (2013). Effect of iron fortification on malaria incidence in infants and young children in Ghana: a randomized trial. JAMA 310, 938-947. doi: 10.1001/jama.2013.277129
Conflict of Interest Statement: The authors declare that the research was conducted in the absence of any commercial or financial relationships that could be construed as a potential conflict of interest.

Received: 12 March 2014; paper pending published: 29 April 2014; accepted: 11 May 2014; published online: 30 May 2014.

Citation: Spottiswoode N, Duffy PE and Drakesmith H (2014) Iron, anemia and hepcidin in malaria. Front. Pharmacol. 5:125. doi: 10.3389/fphar.2014.00125

This article was submitted to Drug Metabolism and Transport, a section of the journal Frontiers in Pharmacology.

Copyright (c) 2014 Spottiswoode, Duffy and Drakesmith. This is an open-access article distributed under the terms of the Creative Commons Attribution License (CC BY). The use, distribution or reproduction in other forums is permitted, provided the original author(s) or licensor are credited and that the original publication in this journal is cited, in accordance with accepted academic practice. No use, distribution or reproduction is permitted which does not comply with these terms. 